\title{
RÉCIPR'OCAL-SPACE AND REAL-SPACE ANALYSES OF COMPOSITIONAL MODULATION IN InAs/AlAs SHORT-PERIOD SUPERLATTICES
}

\author{
D. M. FOLLSTAEDT*, S. R. LEE*, J. L. RENO*, E. D. JONES*, R. D. TWESTEN**, \\ and J. MIRECKI MILLUNCHICK **** \\ *Sandia National Laboratories, M.S. 1056, Albuquerque, NM 87185-1056 \\ **University of Illinois, Urbana, IL 61801-2985 \\ ***National Renewable Energy Laboratory, Golden, CO 80401-3393 \\ ****University of Michigan, Ann Arbor, MI 48109-2136
} A. G. NORMAN***, S. P. AHRENKIEL***, H. R. MOUTINHO***, A. MASCARENHAS***

\section{ABSTRACT}

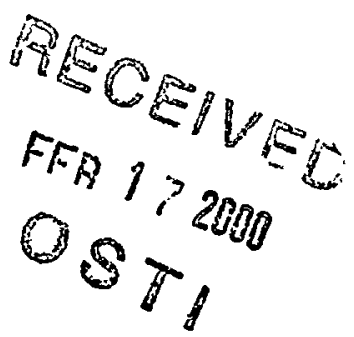

The microstructure of lateral composition modulation in InAs/AlAs superlattices grown by MBE on InP is examined. The use of $x$-ray diffraction, TEM, AFM, and STEM to characterize the modulations is discussed. Combining the information from these techniques gives increased insight into the phenomenon and how to manipulate it. Diffraction measures the intensity of modulation and its wavelength, and is used to identify growth conditions giving strong modulation. The TEM and STEM analyses indicate that local compositions are modulated by as much as 0.38 InAs mole fraction. Plan-view images show that modulated structures consists of short $(\leqslant 0.2 \mu \mathrm{m})$ In-rich wires with a 2D organization in a (001) growth plane. However, growth on miscut substrates can produce a single modulation along the miscut direction with much longer wires $(\gtrsim 0.4 \mu \mathrm{m})$, as desired for potential applications. Photoluminescence studies demonstrate that the modulation has large effects on the bandgap energy of the superlattice.

\section{INTRODUCTION}

Alloying of semiconductors allows their band structures to be tailored to achieve desired properties and permits lattice matching in heterostructures. However, the alloy constituents often have very different electronic bonding and atomic sizes, resulting in thermodynamically immiscible alloys [1,2]. These differences can also produce compositional inhomogeneities during epitaxial growth of alloys. We are interested in the particular example of lateral compositional modulation (CM) that forms during growth of a two-component, vertical shortperiod superlattice (SPS) [3]. In spite of laterally uniform deposition by molecular beam epitaxy (MBE), these materials separate into regions that alternate laterally in enrichment between the two components, as indicated schematically in Fig. 1a. To grow usable materials, this lateral composition modulation needs to be controlled: eliminated where not wanted, or manipulated to form new useful structures. Our intention is to exploit composition separation and produce regular, self-organized structures with nanometer sizes [4]. When the electronic carriers are confined to such sizes in one or two dimensions, quantum confinement effects alter their energies, allowing new properties to be attained. We envision such modulated structures to be potentially useful for semiconductor lasers, solar cells, and polarized light emitters and detectors.

To obtain basic structural properties of the modulated SPS, $\mathrm{x}$-ray diffraction (XRD) is used to detect and analyze additional "satellite" reflections in reciprocal space that result from modulation, as indicated schematically in Fig. 1b. We examine the microstructures in greater detail by using transmission electron microscopy (TEM) to image directly the compositional separations and their organizations within the SPS. Atomic force microscopy (AFM) is also able to detect the lateral modulations by grooves on the specimen surface that indicate the boundaries 


\section{DISCLAIMER}

This report was prepared as an account of work sponsored by an agency of the United States Government. Neither the United States Government nor any agency thereof, nor any of their employees, make any warranty, express or implied, or assumes any legal liability or responsibility for the accuracy, completeness, or usefulness of any information, apparatus, product, or process disclosed, or represents that its use would not infringe privately owned rights. Reference herein to any specific commercial product, process, or service by trade name, trademark, manufacturer, or otherwise does not necessarily constitute or imply its endorsement, recommendation, or favoring by the United States Government or any agency thereof. The views and opinions of authors expressed herein do not necessarily state or reflect those of the United States Government or any agency thereof. 


\section{DISCLAIMER}

Portions of this document may be illegible in electronic image products. Images are produced from the best available original document. 
of the enriched zones. Our studies with real-space images complement the XRD studies; together they produce a greater understanding of the modulated structures. The techniques also provide checks on each other and are found to be quite consistent, as seen with examples below.

Here, we discuss the growth of modulated $(\operatorname{InAs})_{n} /(\mathrm{AlAs})_{\mathrm{m}}$ SPS structures on InP substrates by MBE and their evaluation by these techniques. The microstructures have become well characterized and provide key information about the factors leading to modulation. Comparisons between specific growth conditions allow us to identify the factors controlling $\mathrm{CM}$, and thereby support development of a theoretical understanding of its formation during epitaxial growth. Our results show that modulation amplitudes in this system are large, tenths of InAs mole fraction. We also demonstrate how the modulation can be manipulated to form more ideal geometries, and that modulation has profound effects on the optical emission spectrum of the SPS.

\section{EXPERIMENTAL TECHNIQUES}

\section{Molecular Beam.Epitaxy}

Specimens were grown on epi-ready wafers of (001) InP after oxide desorption in situ under an As flux. Growth rates were calibrated before deposition using reflection high-energy electron diffraction (RHEED) intensity oscillations. A lattice-matched alloy buffer layer of composition $\mathrm{In}_{0.52} \mathrm{Al}_{0.48} \mathrm{As}$ is first grown on the InP substrate to a thickness of 100 or $200 \mathrm{~nm}$. Growth conditions for lattice matching are checked by $\mathrm{XRD}$, but in some specimens isolated dislocations are found extending from this interface to the surface. In much of our early work, the alloy is grown at a rate of $0.7 \mathrm{ML} / \mathrm{s}$ under As-rich conditions. The component layers of the superlattice are then grown at $0.35 \mathrm{ML} / \mathrm{s}$, or at half the alloy rate because only one Group III effusion cell is open at any time instead of two as for the alloy. Since a growth rate study (discussed below) indicates increased modulation intensity at slower growth rates, our latest work uses $0.55 \mathrm{ML} / \mathrm{s}$ and $0.25-0.30 \mathrm{ML} / \mathrm{s}$, respectively. Many specimens are grown with $\mathrm{n} \approx \mathrm{m} \approx 1.5-2$, for which strong CM is observed. In most specimens, the SPS structure contains 100 periods. The SPS structures with $n>2.1$ appear to exhibit 3D growth (islanding) and are not discussed here. The wafers are rotated during MBE to insure radial uniformity of the deposited layers.

The temperature in most of our early work was determined by heating the wafer to a steady $530^{\circ} \mathrm{C}$ as measured by an optical pyrometer and then growing the alloy and SPS. The pyrometer cannot be used to monitor temperature during growth due to thin film optical interference effects. We have since implemented an optical absorption technique that measures the temperature using the shift of the substrate's absorption edge. Since the light is transmitted through the substrate, optical interference effects from the growing layer do not affect the temperature measurement. Continuous monitoring during growth allows a constant temperature to be maintained. A comparison between these two approaches indicates that specimens grown by the first method actually reach a temperature of $\approx 540^{\circ} \mathrm{C}$ during the SPS growth. An actual temperature of $545^{\circ} \mathrm{C}$ appears to be near optimum for strong CM, as determined from XRD (discussed below); this value was maintained by the optical absorption technique in specimens for our recent studies of $\mathrm{CM}$ on miscut substrates, discussed below. Examination of a temperature series by TEM (also below) indicates that a growth temperature of $520^{\circ} \mathrm{C}$ is insufficient to sustain composition modulation. As an upper temperature limit, In thermal desorption becomes appreciable at about $560^{\circ} \mathrm{C}$, making precise control of InAs content unreliable. We conclude that the temperature interval for studying $\mathrm{CM}$ is 520 to $550^{\circ} \mathrm{C}$, with $545^{\circ}$ being near optimum for strong modulation. 
Figure 1a shows a schematic representation of the microstructure of CM in a SPS. In this idealized model, modulation is in the [110] direction with vertical sheets enriched in In or $\mathrm{Al}$ extending uniformly along the [1-10] direction. This 1D lateral modulation produces one or more satellite reflections along [110] on either side of the fundamental reciprocal lattice points of the $(\operatorname{InAs})_{\mathrm{n}} /(\mathrm{AlAs})_{\mathrm{m}}$ SPS, as indicated in Fig. 1b. The alternating layers of the SPS also produce vertical satellites along the [001] direction. Because the average concentration and lattice constant of the coherently strained SPS may differ from those of the lattice-matched buffer and substrate, the SPS reflections and their satellites may be shifted vertically. The upward shift in Fig. $1 b$ represents a SPS in global tension with respect to the buffer and substrate. There is no lateral shift of the fundamental SPS reflections with respect to those of the substrate because the layer is coherent and has the same average lattice constant in the (001) growth plane.

These satellite structures can be used to quantify the degree of modulation and to evaluate the SPS deposition parameters more rigorously than with MBE growth diagnostics. However, doing so requires that reciprocal space be mapped in a plane around the fundamental reflections of the zincblende lattice. Such mapping is very laborious and time consuming by scanning with conventional diffractometers. Instead, we use a position-sensitive detector and accomplish a two-dimensional scan by sweeping only one angle, reducing the time required by up to two orders of magnitude. Elsewhere, the use of these detectors for mapping reciprocal space [5] and implementing this technique for composition modulation [6] are described in more detail. The satellites around each $(00 \mathrm{n})$ fundamental reflection have the same intensity and information. The (002) reflection was chosen for much of this work because it is less intense, and therefore the lateral CM satellites are proportionally stronger and more readily detected against the diffuse background around the fundamental reflection.

In Fig. 2, two such "k-maps" are shown for specimens without and with lateral CM. The scan selected includes the (002) fundamental reflections of the SPS and substrate and the vertical SPS and lateral CM satellites. Some maps show a streak with reduced intensity passing through the intense substrate reflection because the beam had to be attenuated to prevent damage to the detector. Two features allow evaluation of the superlattice parameters. First, the vertical satellite separation is inversely proportional to the period of the superlattice, $p_{s p s}=n+m$. Second, by approximating the SPS as an alloy of composition $\operatorname{In}_{\mathrm{x}} \mathrm{A} \mathrm{l}_{1-\mathrm{x}} \mathrm{As}$ and using its elastic properties, the vertical shift of the fundamental SPS reflection from that of the substrate gives the nominal average In concentration, $x=n /(n+m)$. From these two assessments, the individual layer thicknesses, $\mathrm{n}$ and $\mathrm{m}$, can be determined.

In Fig. 2b, the existence of a lateral satellite indicates the presence of CM. We use the satellite intensity as a measure of the degree of modulation by integrating the $\mathrm{x}$-ray counts within a window that includes the satellite and excludes the fundamental reflections. A window is also placed at the same position in a scan with no satellite (Fig. 2a), and its counts are subtracted from the satellite intensity to correct for the diffuse background around the fundamental reflections. To compare the modulation intensities of two specimens grown under differing conditions, we normalize the satellite intensities by the thickness of the SPS. The lateral spacing of the CM satellite from the fundamental reflection is inversely proportional to the period of the modulations, $\mathrm{p}_{\mathrm{cm}}$. It is notable that this period remains in the interval $20-30 \mathrm{~nm}$ for a wide range of specimens grown with differing $n, m$, global strain and temperature Note that the vertical SPS satellite of Fig. $2 \mathrm{a}$ is much more intense than that of Fig $2 \mathrm{~b}$ where $\mathrm{CM}$ is present. From this comparison it is apparent that forming lateral modulation disrupts the vertical superlattice; 
nonetheless, a remnant of vertical modulation remains that can often be detected with XRD and in TEM images. Cross-sectional TEM was used to examine the two specimens used in Fig. 2, and also indicated that one specimen had modulations while the other did not [7]. Advantages of $\mathrm{XRD}$ are that specimens can be examined non-destructively and more readily since essentially no specimen preparation is required, whereas TEM specimens require extensive preparation. In addition, $\mathrm{XRD}$ gives a semiquantitative evaluation of the relative strength of $\mathrm{CM}$.

Examinations of modulated $(\mathrm{InAs})_{\mathfrak{n}} /(\mathrm{AlAs})_{\mathrm{m}}$ structures with plan-view TEM in the [001] orientation indicate that they are not composed of simple 1D modulations; rather, they have a 2D organization with two modulation directions in the (001) growth plane [8], as seen in Fig. 3. The idealized case in Fig. $1 \mathrm{~b}$ of one set of lateral satellites along [110] is thus not rigorously satisfied; careful examination of electron diffraction patterns taken in plan-view TEM reveals two sets of lateral satellites around the fundamental reflections [7], as seen in Fig. 3a. For the case of a SPS with moderate tensile strain as in Fig. 3, the modulations occur nearly along the [310] and [130] directions (discussed below), producing satellites at $\approx 26.5^{\circ}$ on either side of the [110] direction. However, when examining the (1-10) plane in reciprocal space with position-sensitive X-ray diffraction, the two satellites are projected onto this plane and detected as a single lateral satellite along [110], much as indicated in Fig. 1b. The actual satellite locations being out of the (1-10) plane was confirmed by their detection with the orthogonal (110) plane [4]. Grazing incidence diffraction would be needed to directly examine the (001) plane in reciprocal space. Thus the ability of TEM to examine the (001) plane more readily provides this additional information needed for a complete understanding of the modulations.

\section{Microscopy Techniques}

Contrast in TEM images is frequently produced by diffraction effects. The specimen is tilted to produce a "two-beam" condition with the transmitted beam and only one strongly diffracting beam in the diffraction pattern. Images then exhibit contrast due to strain gradients along the direction of the reciprocal lattice vector of the diffracted beam, $g$. This approach is quite useful for examining lattice defects, but not for imaging composition variations in a coherent layer. The strain contrast of $\mathrm{CM}$ specimens can be qualitatively understood with the schematic in Fig. 4 depicting the lattice planes of alternating In-rich and Al-rich layers in a thinned cross-section specimen [9]. The $x$-direction is the [110] modulation direction in Fig. 1a. A strongly diffracting $\mathbf{g}=(220)$ reflection produces strain contrast due to the alternations between Al-rich atomic planes with smaller lattice constants and In-rich planes with larger ones. In addition, $\mathbf{g}$ vectors in this direction produce contrast from the relaxation of strains at the surfaces of the cross-sectioned specimen, indicated in Fig. 4 by the curving of lattice planes at the top and bottom. Such images have complex contrasts from which composition variations are not readily identified.

To avoid such strain effects, contrast from a $\mathrm{g}$ vector perpendicular to these strains and along the growth direction needs to be selected; the choice is basically between (002) and (004). The (004) vector is more sensitive to other strain effects (irregularities in the thinned specimen, additional relaxations at the free surface of the SPS layer) among which composition differences must be identified. Just as for XRD, the intensity of the (002) reflection and correspondingly its diffraction contrast effects are much weaker. However, $\{200\}$-type reflections are very sensitive to composition changes in III-V alloys; they are produced by scattering differences between the Group III and Group V sublattices of the zincblende structure, and are absent in Group IV semiconductors where both sublattices are equivalent. Again in mathematical similarity to XRD, the sensitivity to composition changes is highest for $\{002\}$ reflections when normalized to the intensity of the average composition, as in a contrast calculation. 
In the TEM, it is not possible to obtain diffraction conditions with only the (002) reflection excited, but tilting the specimen so that only the (001) systematic row of reflections is diffracting reduces the effect of strain contrast and results in (002) dark-field images that reliably reflect composition variations. The example in Fig. 5 shows the composition variations clearly: In-rich columns are light with dark bands on either side, while the Al-rich regions are approximately a medium gray. The dark bands on either side of the bright In-rich band are due to a null in the $\{200\}$ diffracted intensity. At an average concentration of $\operatorname{In}_{0.52} \mathrm{Al}_{0.48} \mathrm{As}$, the scattering from the Group III sublattice cancels that from the Group V (As) sublattice $[9,10]$. The In-rich columns are thus enriched above the average concentration of $x=0.46$ for this SPS to $x>0.52$. Calculations involving the relative thicknesses of the In-rich column and the wider Al-rich regions, as well as the shape of the In concentration profile (below), give concentrations of $\mathrm{In}_{0.73} \mathrm{Al}_{0.27} \mathrm{As}$ at the center of the columns and $\mathrm{In}_{0.31} \mathrm{Al}_{0.69} \mathrm{As}$ for the broad areas between them.

Cross-sectional TEM imaging was also used to examine the growth-temperature dependence of CM. In the SPS imaged in Fig. 6, the growth was begun at $530^{\circ}$ as controlled by the substrate absorption method and decreased in $10^{\circ} \mathrm{C}$ increments after subsequent intervals of $20 \mathrm{~nm}$. A growth temperature of $530^{\circ} \mathrm{C}$ is just sufficient to initiate $\mathrm{CM}$, as seen by a few low-contrast columns like those indicated. These modulations persist into the $520^{\circ} \mathrm{C}$ interval, but not across it; no modulation is detected at $510^{\circ} \mathrm{C}$. Thus growth temperatures $\geq 530^{\circ} \mathrm{C}$ are required for $\mathrm{CM}$.

For plan-view TEM imaging, a g vector cannot be chosen which is necessarily insensitive to in-plane lattice constant changes and surface relaxation. However, (200) and (020) beams show contrasts as expected for composition variations, apparently with little influence of strain [8]. Figure $3 b$ is a (200) dark-field image showing the lateral organization of composition variations. Once again In-rich wires appear light; higher magnifications show the gray/black/white/black... contrast variation, discussed above as being indicative of large composition variations. The 2D organization of the contrast is apparent, and the two modulation directions are indicated. Many In-rich wires are $\leqslant 0.1 \mu \mathrm{m}$ long, with some up to $0.2 \mu \mathrm{m}$. In some areas wires of one or the other modulation are seen, while in other areas, the two modulations appear to mix and produce small In-rich areas, which appear as dots in plan view but are actually In-rich "pillars" rising up through the $\sim 0.1 \mu \mathrm{m}$-thick SPS. Figure $3 \mathrm{c}$ is a Fourier transform of the dark-field image, which indicates the average of modulations in this structure. The pattern exhibits two bright diffuse peaks of intensity with secondary peaks at twice the radial distance. The angle between these two modulation directions is $53^{\circ}$, as indicated on the figure. These two directions lie on either side of [110], and the angle agrees with that for the [310] and [130] directions [8]. The width of the peaks indicates that modulations occur within a range of angles centered around these directions. Thus, the in-plane organization of the modulations is quite complex. Specimens grown with global compression also show $2 \mathrm{D}$ lateral organization but with modulations along the $\langle 100\rangle$, as discussed by us in an accompanying paper by Norman et al. [11].

Note that SPS modulation can still be detected in Fig. 5 as vertically alternating black/white lines superimposed on the (002) contrast in this strongly modulated specimen. This modulation is imaged because the objective aperture includes a vertical SPS satellite, whose intensity is reduced but is non-zero. At the position of the In-rich bright columns, the SPS shows a slight dip, which has been related to the overall tensile strain of the SPS [4]. The vertical SPS layers represent a frozen "history" of the surface during growth of the SPS, and the dip is present in each layer up to the top of the specimen.

The dip produces a groove along In-rich wires at the top surface, explaining why nonchemically sensitive AFM can be used to detect lateral composition variations by examining surface topography [8]. Shown in Fig. 3d is an AFM image of the surface of the specimen of 
Fig. 3b. A very similar organization of lateral modulation is inferred from the arrangement of grooves on the surface. Two modulation directions nearly along [310] and [130] are indicated on the figure. Thus XRD, electron diffraction, dark-field TEM, and AFM all indicate the same 2D in-plane organization of the modulated structure.

The use of a dedicated Scanning Transmission Electron Microscope (STEM) with a subnanometer electron beam has provided new information on composition profiles along the modulation direction and on local compositions [9]. First, the intensity of electrons diffracted to large angles in a High-Angle Annular Dark-Field (HAADF) image is nearly proportional to the square of the local atomic number [12]. The linescan in Fig. 7 across several modulation periods indicates that the In composition peaks in the center of the columns in Fig. 5, while the Al-rich regions are broader with a more nearly constant composition. This shape was used to interpret the (002) contrast variations discussed above in this section. Second, the characteristic elemental $\mathrm{x}$-rays generated in the microscope when the beam is on enriched areas can be used to obtain local compositions by using $x$-rays generated in the buffer with composition $\operatorname{In}_{0.52} \mathrm{Al}_{0.48} \mathrm{As}$ as an internal calibration. Figure 8 shows $\mathrm{X}$-ray spectra obtained from In-rich and from Al-rich regions. The differences in the $\mathrm{Al} \mathrm{Kx}$-ray peaks and the In $\mathrm{L}$ peaks are sizeable, while the As $\mathrm{L}$ peak amplitude is essentially unchanged. The peak ratios indicate local compositions of $\mathrm{In}_{0.70} \mathrm{Al}_{0.30} \mathrm{As}$ and $\mathrm{In}_{0.43} \mathrm{Al}_{0.57} \mathrm{As}$. These compositions are similar to those derived from contrast considerations. More generally, several TEM-based techniques each indicate that the amplitude of the composition variations is large, tens of percent InAs mole fraction [9].

\section{FUNDAMENTAL INVESTIGATIONS OF GROWTH PARAMETERS}

\section{Superlattice Parameters (n, m)}

A plot summarizing the intensity of $\mathrm{CM}$ as measured with lateral satellite intensities in XRD is given in Fig. 9 for the $(n, m)$ layer combinations studied by us. The area of the data points is proportional to the intensity of the satellite. Thicknesses of InAs layers were limited to $n \leq 2.1$ to maintain two-dimensional growth. Several trends are indicated in this plot. First, the specimens with non-zero intensity lie between the boundaries $0.43 \leq x \leq 0.63$ [6]. Alternatively, these limits can be expressed as the in-plane strain of the SPS average lattice constant when fitted coherently to the substrate; the boundaries are then $|\varepsilon| \leq 0.007$. The most intense modulations are found near the lattice-matched condition of $x=0.52$. An early TEM investigation of a subset of these specimens that spanned this range showed changes in the contrast of (002) dark-field images and in the regularity of spacing of In-rich columns that reflected this trend [13]. This agreement establishes the integrated satellite intensity as an accurate measure of the CM intensity.

The most intensely modulated specimens occur for $\mathrm{n} \gtrsim 1.7$. This thickness is close to that found by others $[14,15]$ for InAs island formation on $\operatorname{In}_{0.52} \mathrm{Al}_{0.48} \mathrm{As}$ buffers on $\operatorname{InP}$ substrates. InAs island formation is expected to be promoted further by the In segregation observed at the surface of the growing buffer layer [9]. The bright line at the interface between the buffer and SPS (arrowed) in Fig. 7 indicates that the first InAs layer contains more In that subsequent ones. Cross-section images in Figs. 6 and 11 show an enriched first layer as well. Moreover, planview TEM images of a single layer of 1.9 ML on InAs deposited on the buffer show some small islands that are nanometer-sized dots or elongated wires [11]. A plausible inference then is that $\mathrm{CM}$ requires exceeding a threshold value of $\mathrm{n}$ in order to produce islands that then initiate the formation of In-rich columns above them. Some cross-section TEM images show evidence for such an island in the first InAs layer of the SPS. 
We grew several specimens to test this hypothesis. In the lower right side of the plot are two points labeled 1 and 2 . Point 1 , from the early set of data, has near-zero modulation intensity and lies on the lower composition boundary. We grew specimen 2 , with $n=2.1$ and $m=0.95$, to also be outside this boundary but with increased $\mathrm{n}$. This specimen also showed no modulation intensity, which argues against the failure of point 1 to achieve a critical InAs thickness, but is consistent with being outside the $\mathrm{x}=0.63$ boundary discussed above. Point 3 lies at the other side of the composition bounds and specimen 4 was subsequently grown with $n=1.8$ and $m=2.7$. Again, the increase in $\mathrm{n}$ did not cause $\mathrm{CM}$ to form. Finally, we grew specimen 5, which lies near $\mathrm{x}=0.52$ and is well within the composition bounds, but has $\mathrm{n}=1.55$. Its modulation is greatly reduced, which could be consistent with a critical thickness. It thus appears that InAs island formation may initiate CM, but the development of CM throughout the SPS layer is determined by the average SPS composition being within the indicated limits $[6,13]$.

Another trend indicated is that intense modulations are found for superlattice period values $(n+m)=3$ to $4[6,7]$. Several specimens in the plot were grown with $n \approx m$ and are near $x=$ 0.52 as expected for strong modulation. However, the intensity is seen to decrease for values of $(n+m)$ near 2 and is essentially absent for 1 , even though their compositions place these specimens within the boundaries noted above. Again, TEM characterization of these specimens is consistent with the satellite intensities [6,7]. This dependence on modulation period and the absence of strong CM in our alloy buffer layers indicate the importance of the superlattice deposition scheme for producing CM. Perhaps when $n, m \leqslant 1$, the superlattice layers interdiffuse during deposition and become more like a random alloy that does not produce strong lateral modulation. For our current specimens, $(n+m)=3$ to 4 is found to produce strong modulation; we hope to examine larger values of the superlattice period in future work.

\section{Growth Rate}

The effects of growth rate on $\mathrm{CM}$ were investigated for growth at $\approx 540^{\circ} \mathrm{C}\left(530^{\circ} \mathrm{C}\right.$ at start of growth as determined by optical pyrometry; see above), with $\mathrm{n}$ and $\mathrm{m}$ chosen to produce slight tension for optimum modulation intensity. The SPS were grown with average rates of $0.50,0.36$, 0.25 and $0.18 \mathrm{ML} / \mathrm{s}$; the lowest rate was attained by using the same conditions as for $0.36 \mathrm{ML} / \mathrm{s}$ but with growth interrupts (MBE shutters closed) after each superlattice layer to produce a 50\% duty cycle. The modulation intensity was monitored using satellites around the $\mathbf{k}=(224)$ fundamental reflection, as seen in Fig. 10. Because this reciprocal lattice vector has a component in the (001) plane and along the modulation direction, an asymmetric satellite distribution occurs as discussed in [6]. Satellite intensities for all four specimens were found to be strong, but that for the lowest growth rate is $\sim 40 \%$ more intense than that for the fastest rate. This indicates that kinetics play an important role in the development of modulation, which is consistent with our growth temperature studies.

The satellites also indicate that the vertical organization of the $\mathrm{CM}$ is affected by growth rate. In Fig. 10, the vertical full width at half maximum of the satellite is compared for growths at 0.50 and $0.18 \mathrm{ML} / \mathrm{s}$. The width is clearly much broader for the slower growth, although the integrated intensity is higher. The width decreases continuously with increasing growth rate for all four specimens. This width in reciprocal space is inversely proportional to the domain height over which composition modulation is vertically correlated; values are indicated in the Fig. 10 . Cross-section TEM images from the same specimens [7] are shown in Fig. 11 and provide some support for this interpretation. The In-rich columns illuminated in (002) dark-field images extend across most of the $100 \mathrm{~nm}$-thick SPS for the fast growth rate (Fig. 11a), but only over a more limited distance for the slow growth rate. In both images, the illuminated column heights 
are comparable to the indicated XRD domain heights. The reduced height could indicate that the column terminated or wandered laterally out of the cross-section plane being examined. Thus a slower growth rate at a temperature $\approx 540^{\circ} \mathrm{C}$ enhances the magnitude of $\mathrm{CM}$ but degrades its vertical organization.

\section{$\underline{\text { Substrate Miscut }}$}

The growth on oriented (001) InP substrates has always been found to produce a 2D organization of compositionally enriched wire structures, as seen in Fig. 3b [7;8]. A single modulation direction and long wires are desirable, since a regular, self-organized structure exhibiting $1 \mathrm{D}$ quantum confinement of carriers in long wires may be useful for manipulating electronic properties and for implementing them in new devices. Such a structure is likely to exhibit strong anisotropies in the growth plane for properties such as polarized photon emission, conductivity and carrier mobilities. In an attempt to achieve this microstructure, we grew specimens on miscut substrates. Figure 12a is an (020) dark-field, plan-view TEM image showing the modulated structure of an SPS grown on (001) InP that was miscut $2^{\circ}$ toward (101). The [310] modulation direction is now dominant in the image and wire lengths are often $0.3 \mu \mathrm{m}$ long, with some up to $0.4 \mu \mathrm{m}$. This is a dramatic change over the microstructure seen in Fig. 3 for growth on oriented (001) InP. Moreover, the modulation intensity remains strong, as indicated by the gray/black/white/black... TEM contrast and the large composition variations found in this specimen by STEM: from $\operatorname{In}_{0.70} \mathrm{Al}_{0.30} \mathrm{As}$ to $\operatorname{In}_{0.43} \mathrm{Al}_{0.57} \mathrm{As}$ (see Fig. 8).

We can interpret the selection of the [310] modulation by the miscut in terms of surface steps. The schematic in Fig. $12 \mathrm{~b}$ shows how the steps align with respect to the modulations and wires. The average step direction is misaligned only $18^{\circ}$ with respect to the wires of the [310] modulation, whereas it is $72^{\circ}$, or nearly perpendicular, to the wires of the [130] modulations. Other studies of modulation in a compressively strained SPS, which has a different 2D organization for growth on (001), also respond to miscutting toward (101) by enhancing the modulation with wires that are near-parallel to the steps [11]. The step edges may. alter the organization of $\mathrm{CM}$ is several ways. For instance, step edges may strain the lattice to enhance the attachment of one species over another. Surface diffusivities may also become anisotropic due to a preferred edge direction. The anisotropy in surface step density may also affect the modulation direction through step bunching and InAs island nucleation, as discussed in [11]. From the dramatic change in wire length and modulation character, it is clear that the use of miscut substrates will play an important role in manipulating the microstructure of $\mathrm{CM}$ in this alloy system. A regular 1D modulation and long wires are also achieved with miscut substrates and SPS layers grown in global compression [11].

\section{OPTICAL EMISSION}

An important motivation for our investigations is to develop modulated structures with new electronic properties. The intense CM in our materials indeed changes the low-temperature photoluminescence (PL) emission energy of the SPS layer greatly. The PL spectrum from the miscut specimen with strong modulation (see Figs. 8 and 12) is shown in Fig. 13. This specimen has $\mathrm{x} \approx 0.5$, for which emission is expected to occur at $1.58 \mathrm{eV}$; instead, the emission is observed at $1.07 \mathrm{eV}$. This large reduction, $0.5 \mathrm{eV}$, is found for our most strongly modulated specimens [16]. Moreover, the amount of the reduction was found to track the intensity of satellite reflections across the composition range for which they are detected with $\mathrm{XRD}, 0.43<\mathrm{x}<0.62$, indicating that the reduced PL energy is indeed determined by the CM. 
For specimens with strong CM, the SPS structure is disrupted (see Fig. 2) and is expected to have little effect on the bandgap emission energy. Since the bandgap offsets between InAs-rich alloys and AlAs-rich alloys are Type I, the PL emission will occur from the In-rich regions and to a first approximation, will be determined by the bandgap of the locally enriched alloy. Compositional analysis with EDS indicates $\mathrm{x}=0.70$ for the In-rich regions of this specimen (see Fig. 8), for which the known bandgap dependence [17] predicts an emission energy of $1.09 \mathrm{eV}$. This close agreement with the observed energy supports the simplified interpretation used here, which ignores other effects on emission energy such as coherency strain [16]. Similarly good agreement was found by us previously for a different specimen for which the local In-rich composition had been determined by EDS [18]. The observed PL energies are also found to compare favorably with a theoretical treatment of bandgap reduction by Zhang and Mascarenhas [19] when CM amplitudes determined with TEM-related techniques are used.

\section{CONCLUSIONS}

Superlattices of $\operatorname{InAs_{n}} / \mathrm{AlAs}_{\mathrm{m}}$ grown on InP can exhibit strong lateral composition modulation. We have demonstrated how the modulation can be characterized with XRD, TEM, STEM and AFM. Detecting satellites in reciprocal space with XRD provides a convenient way to assess semiquantitatively the modulation intensity, and to evaluate the effects of changes in growth parameters. The real-space microscopy techniques validate the XRD method for assessing modulation and provide detailed microstructural information; they also complement the XRD information by enabling imaging of the (001) plane in plan-view.

Our structural techniques have identified the conditions necessary for growth of SPS structures with strong modulation. A temperature of $545^{\circ} \mathrm{C}$ appears optimum, while slowing the growth rate increases modulation at the cost of reduced vertical organization. The study of superlattice parameters ( $\mathrm{n}$ and $\mathrm{m}$, Fig. 9) suggest that two criteria are required for intense modulation: 1) adequate layer thicknesses, $\mathrm{n}, \mathrm{m}>1.5$, and 2) appropriate average concentration, $0.43<x<0.63$. Criterion 1) produces strong modulation, but smaller thicknesses nonetheless produce weak modulation. Criterion 2) appears more nearly absolute; modulation is not observed outside these composition boundaries. The first criterion may be related to InAs island formation. The second criterion gives compositions requirements for lateral decomposition to occur and propagate, and is likely have its origins in the energetics and thermodynamics of bonding new atoms onto the growing surface. The composition requirements may be related to limitations imposed by global strain on organizing the compositionally enriched wires into a $2 \mathrm{D}$ pattern in the growth plane. The difference in 2D patterns of tensile and compressive SPS layers is perhaps an indication that global strain plays a role.

Miscut substrates appears very useful for manipulating the growth-plane organization of composition modulation into a regular 1D structure with long wires. These structures may produce quantum confinement of carriers in a geometrical arrangement that appears more usable for devices. The anisotropy in the growth plane is likely to produce a preferred polarization to optical emission that might be useful for semiconductor lasers, and a differing conductivity along the wire direction from that perpendicular to it. Composition modulation produces dramatic effects on the band structure and optical emission of the InAs/AlAs superlattices. Combining them with the more ideal 1D modulation structure may allow new applications to be achieved. In particular, the ability to emit or absorb light at energies different from that for lattice-matching to the substrate is a new degree of freedom in bandgap engineering that can be exploited. 


\section{ACKNOOWLEDGEMENTS}

The authors wish to thank L. L. Stephenson for assistance with the MBE specimen growth, M. P. Moran for support in obtaining TEM images, and R. L. White for assistance with obtaining PL spectra. This work is supported by Division of Materials Sciences, Office of Basic Energy Sciences of the U. S. Department of Energy. Sandia is a multiprogram laboratory operated by Sandia Corporation, a Lockheed Martin Company, for the U. S. Department of Energy under contract DE-AC04-94A185000. NREL is a national laboratory of the U. S. Department of Energy operated by the Midwest Research Institute, Battelle, and Bechtel. Work at the University of Illinois was done in the Center for Microanalysis of Materials under U. S. Department of Energy grant DE-FG02-91ER45439.

\section{REFERENCES}

1. J. W. Cahn, Acta Metall. $\underline{9}, 795$ (1961).

2. G. B. Stringfellow, J. Cryst. Growth $\underline{\mathbf{5 8}}, 194$ (1982); $\underline{\mathbf{6 5}}, 454$ (1983).

3. S. T. Chou, K. Y. Cheng, L. J. Chou and K. C. Hsieh, Appl. Phys. Lett. 66, 2220 (1995).

4. J. Mirecki Millunchick, R. D. Twesten, S. R. Lee, D. M. Follstaedt, E. D. Jones, S. P. Ahrenkiel, Y. Zhang, H. M. Cheong and A. Mascarenhas, MRS Bulletin 22 (7), 38 (1997).

5. S. R. Lee, B. L. Doyle, T. J. Drummond, J. W. Medernach and R. P. Schneider Jr., in "Advances in x-ray Analysis, Vol. 38", eds. P. Predecki et a.l, (Plenum Press, NY, 1995) p. 201.

6. S. R. Lee, J. Mirecki Millunchick, R. D. Twesten, D. M. Follstaedt, J. L. Reno, S: P. Ahrenkiel and A. G. Norman, J. Materials Science: Materials In Electronics 10, 191 (1999).

7. S. P. Ahrenkiel, A. G. Norman, M. M. Al-Jassim, A. Mascarenhas, J. Mirecki Millunchick, R. D. Twesten, S. R. Lee, D. M. Follstaedt and E. D. Jones, J. Appl. Phys. 84, 6088 (1998).

8. A. G. Norman, S. P. Ahrenkiel, H. Moutinho, M. M. Al-Jassim, A. Mascarenhas, J. Mirecki Millunchick, S. R. Lee, R. D. Twesten, D. M. Follstaedt, J. L. Reno and E. D. Jones, Appl. Phys. Lett. 73, 1844 (1998).

9. R. D. Twesten, D. M. Follstaedt, S. R. Lee, E. D. Jones, J. L. Reno, J. Mirecki Millunchick, A. G. Norman, S. P. Ahrenkiel and A. Mascarenhas, Phys. Rev. B 뜨, 13619 (1999).

10. R. D. Twesten, J. Mirecki Millunchick, S. P. Ahrenkiel, Y. Zhang, S. R. Lee, D. M. Follstaedt, A. Mascarenhas and E. D. Jones, Mat. Res. Soc. Symp. Proc. 441, 187 (1997).

11. A. G. Norman, S. P. Ahrenkiel, H. R. Moutinho, C. Baliff, M. M. Al-Jassim, A. Mascarenhas, D. M. Follstaedt, S. R. Lee, J. L. Reno, E. D. Jones, J. Mirecki Millunchick, and R. D. Twesten, elsewhere these proceedings.

12. A. Howie, J. Microsc. 117, 11 (1979).

13. D. M. Follstaedt, R. D. Twesten, J. Mirecki Millunchick, S. R. Lee, E. D. Jones, S. P. Ahrenkiel, Y. Zhang and A. Mascarenhas, Physica E 2, 325 (1998).

14. H. Li, J. Wu, B. Xu, J. Liang and Z. Wang, Appl. Phys. Lett. 72, 2123 (1998).

15. J. Brault, M. Gendry, G. Grenet, G. Hollinger, Y. Desières and T. Benyattou, Appl. Phys. Lett. $\underline{73}, 2932$ (1998). 
16. E. D. Jones, D. M. Follstaedt, S. R. Lee, J. L. Reno, J. Mirecki Millunchick, S. P. Ahrenkiel, A. Mascarenhas, A. G. Norman, Y. Zhang and R. D. Twesten, Thin Solid Films, $\underline{357}, 31$ (1999).

17. $\mathrm{E}=3110-3395 \mathrm{x}+725 \mathrm{x}^{2}$ (in meV); I. J. Fritz, private communication, 1998.

18. R. D. Twesten, D. M. Follstaedt, J. Mirecki Millunchick, S. R. Lee, A. G. Norman, S. P. Ahrenkiel, J. L Reno, E. D. Jones, A. Mascarenhas, and Y. Zhang, Microscopy and Microanalysis $\underline{\mathbf{5}}$ (supplement 2: Proceedings), 174 (1999).

19. Y. Zhang and A. Mascarenhas, Phys. Rev. B $\underline{\mathbf{5 7}}, 12245$ (1998). 


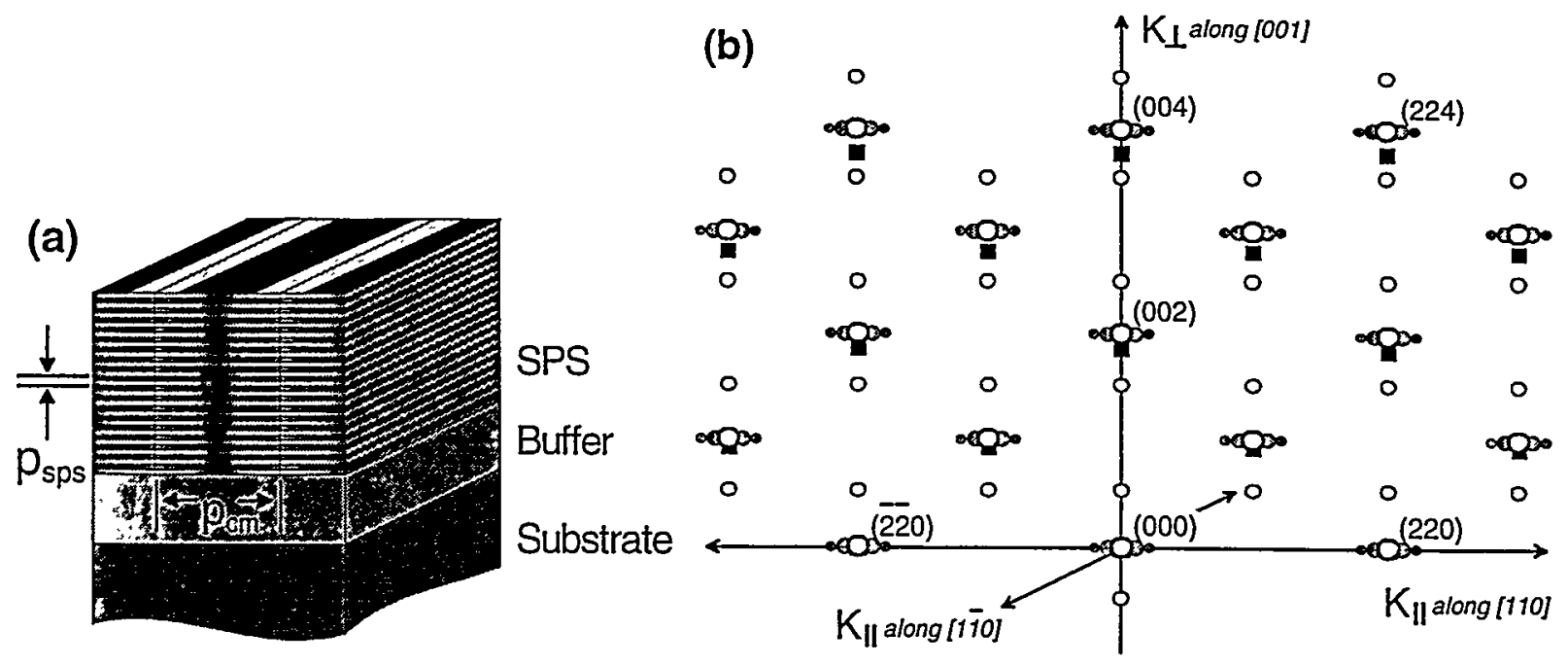

Figure 1. a) Real-space structure of an SPS with 1D lateral CM. b) Corresponding reciprocal space structure. Black squares represent the reciprocal lattice of the InP substrate and latticematched $\mathrm{In}_{0.52} \mathrm{Al}_{0.48} \mathrm{As}$ buffer, and white circles represent the lattice of a coherently strained alloy with the average composition of the SPS. Light gray circles along [001] represent first-order SPS satellites, and dark gray circles along [110] represent transverse satellites due to CM. 


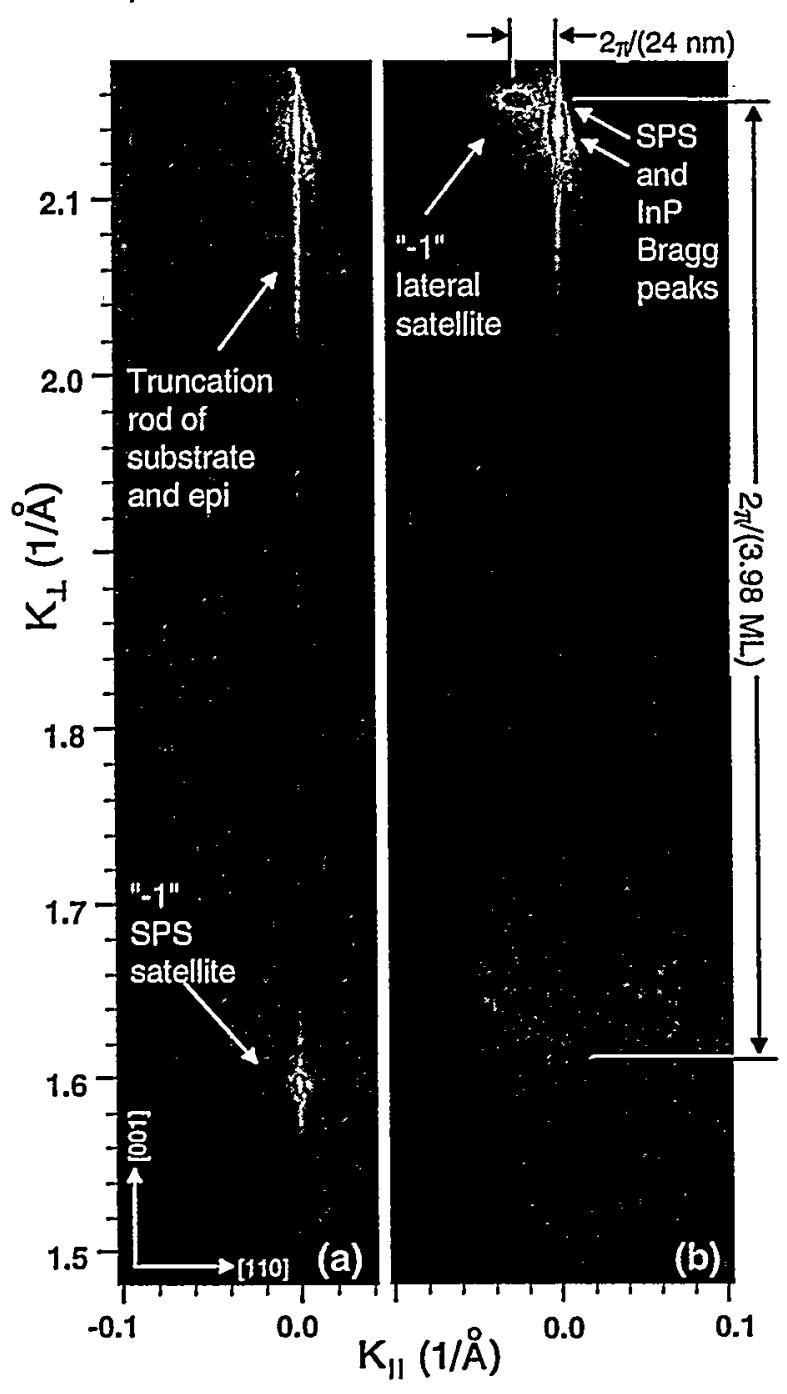

Figure 2 (above). Reciprocal space maps around (002). a) $\mathrm{n}=1.60, \mathrm{~m}=2.18, \mathrm{x}=0.422$, showing no $\mathrm{CM}$ (sample \#EA0121). b) $\mathrm{n}=$ $1.85, \mathrm{~m}=2.13, \mathrm{x}=0.466, \mathrm{p}_{\mathrm{cm}}=24 \mathrm{~nm}$, showing strong $\mathrm{CM}$ (EA0122).

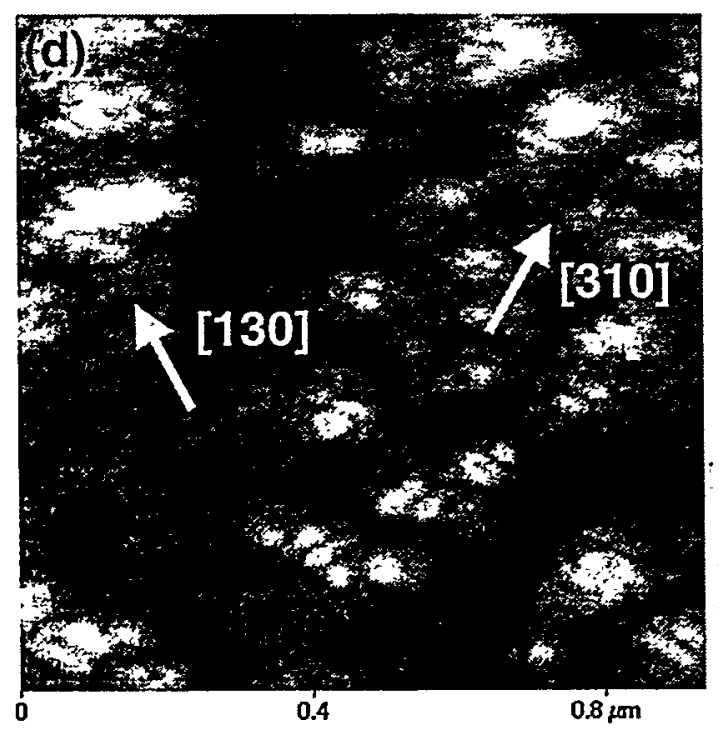

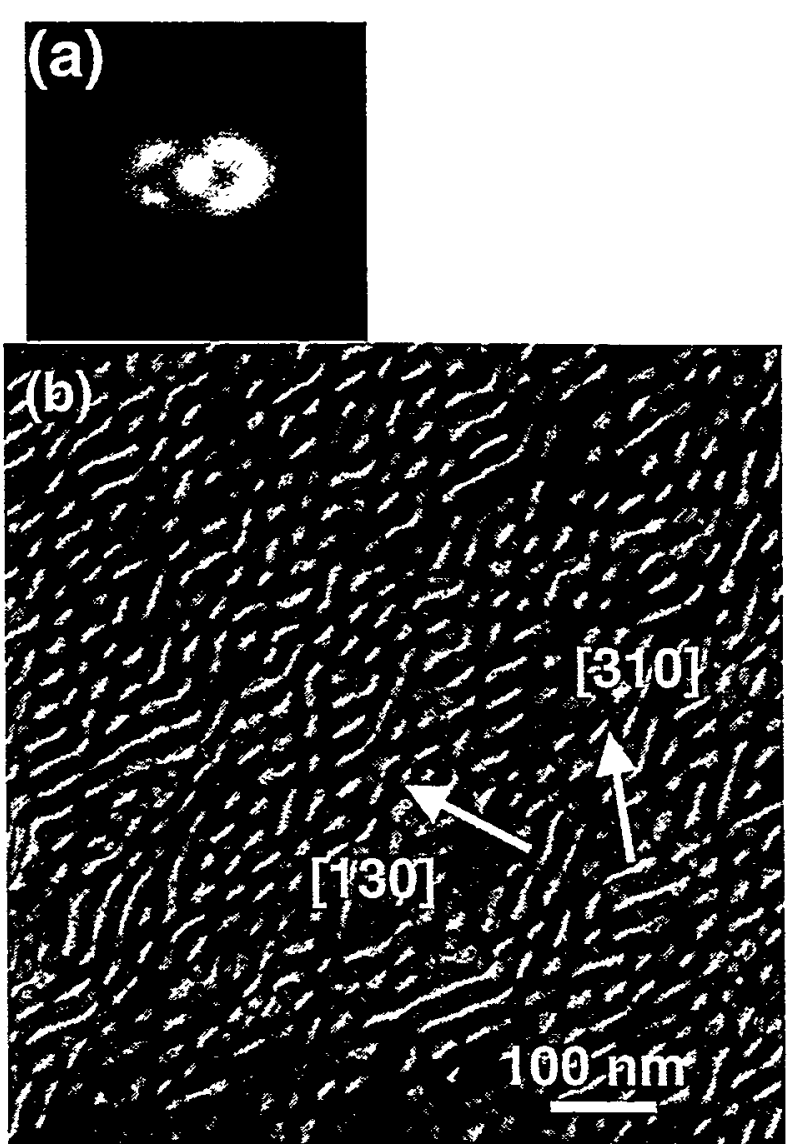

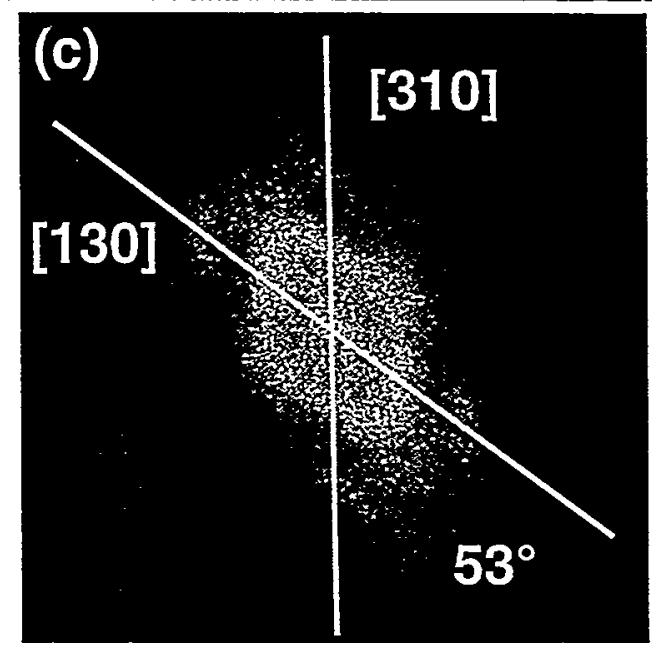

Figure 3. Above: a) Enlarged electron diffraction image of (020) reflection taken in [001] plan view, showing 2D satellite structure (EA0117). b) Plan-view (020) dark-field image showing 2D organization of wires and two modulations (arrows) along [310] and [130]. $\mathrm{n}=\mathrm{m}=1.95$ (EA0479). c) Fourier transform of dark-field image exhibiting diffuse peaks along two modulation directions. At left: d) AFM image of same specimen showing grooves on the SPS surface with the same modulation directions and in-plane organization. 


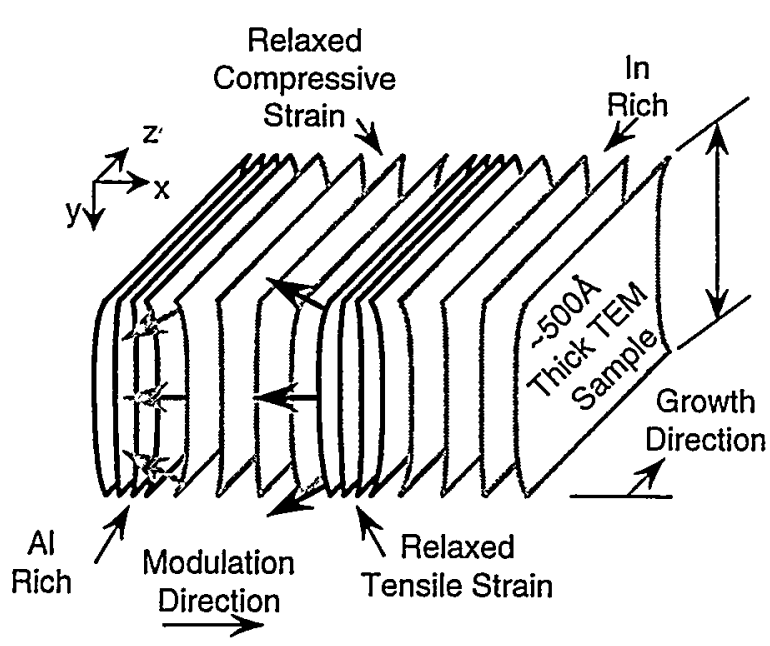

Figure 4. Schematic of atomic planes in CM material, showing strain along the modulation direction, and strain relaxation at the surfaces of thinned cross-sectional TEM specimens.

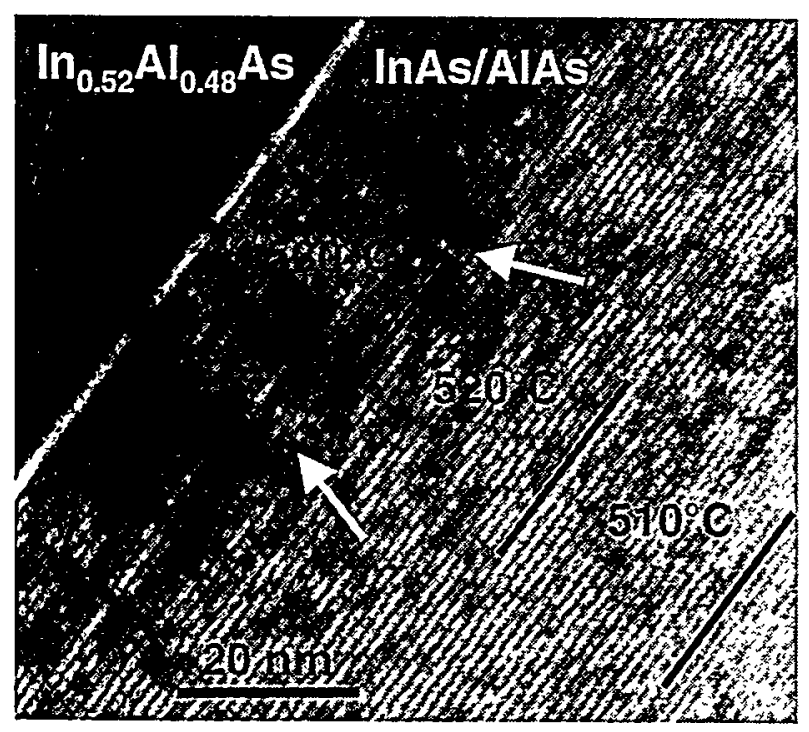

Figure 6. Cross-sectional (002) dark-field image of SPS with $\mathrm{n}=1.94, \mathrm{~m}=2.08$, showing weak modulations (arrowed) during initial growth at $530^{\circ} \mathrm{C}$, which do not persist in a subsequent $20 \mathrm{~nm}$ interval grown at $520^{\circ} \mathrm{C}$, and are not found during growth at $510^{\circ} \mathrm{C}$ (EA0321).

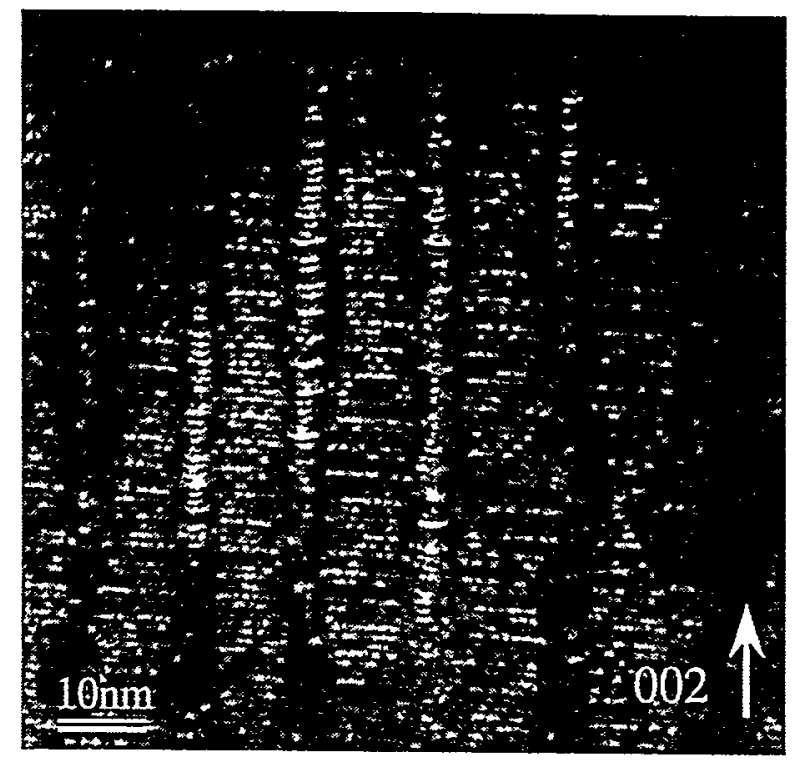

Figure 5. Cross-sectional, (002) dark-field TEM image showing vertical In-rich columns (light) with dark bands on either side due to a null in the structure factor at a composition of $\mathrm{In}_{0.52} \mathrm{Al}_{0.48} \dot{A} \mathrm{~s}$ (MS511H). 

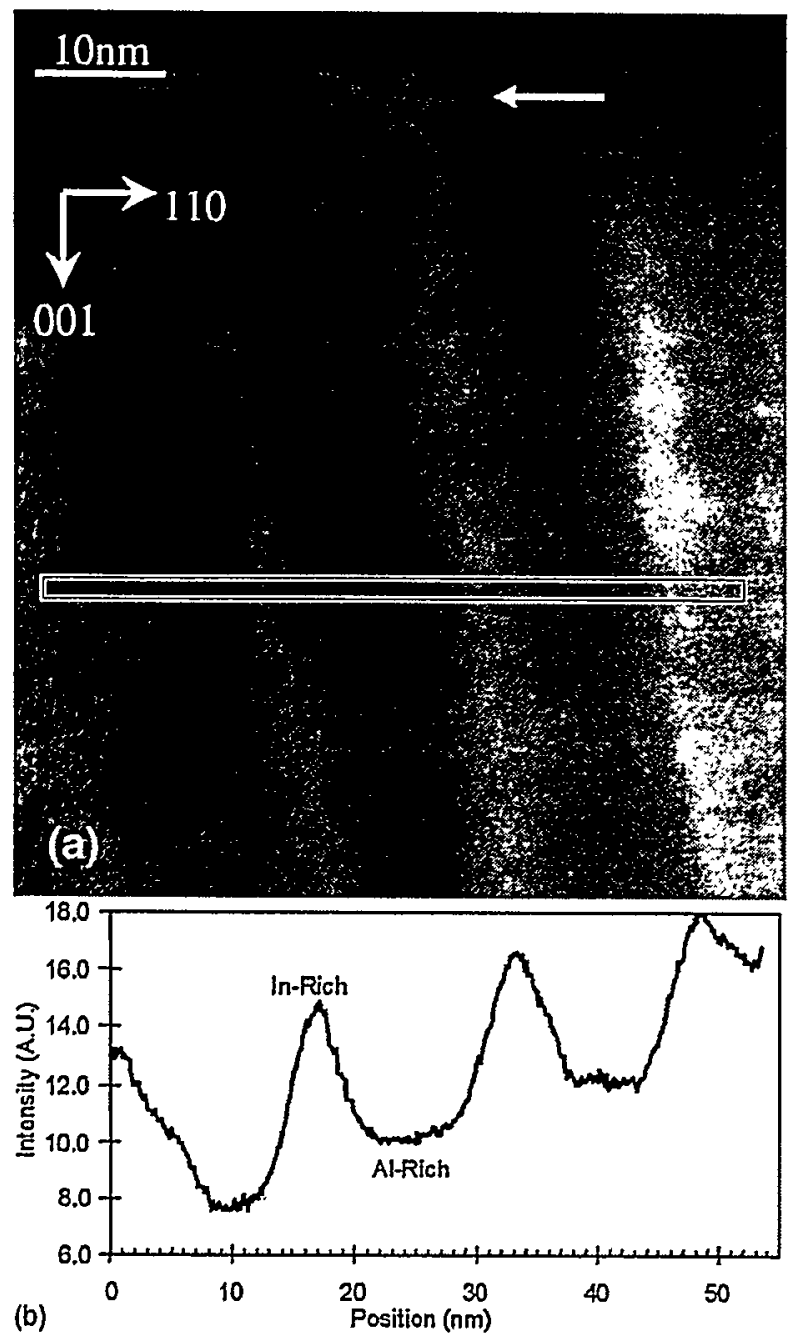

Figure 7. a) HAADF cross-sectional image of modulated SPS with $\mathrm{n}=1.86, \mathrm{~m}=2.13$, taken with STEM instrument showing bright In-rich columns. Arrow at bright interface with buffer shows that first InAs layer contains more In. b) Intensity linescan across In-rich columns at position indicated in a). (EA0122).

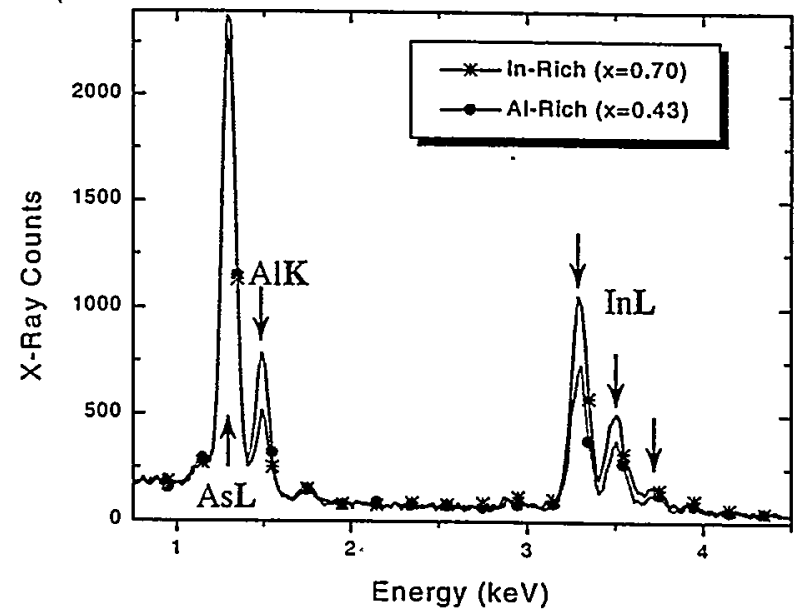

Figure 8. Energy dispersive $\mathrm{x}$-ray spectra taken with STEM from In-rich and Al-rich regions. Note that the As-L intensity remains constant while those of $\mathrm{Al}-\mathrm{K}$ and In-L vary between the two positions, giving the stated compositions. $\mathrm{n} \approx 1.95, \mathrm{~m} \approx 1.95$ (EA0478). 


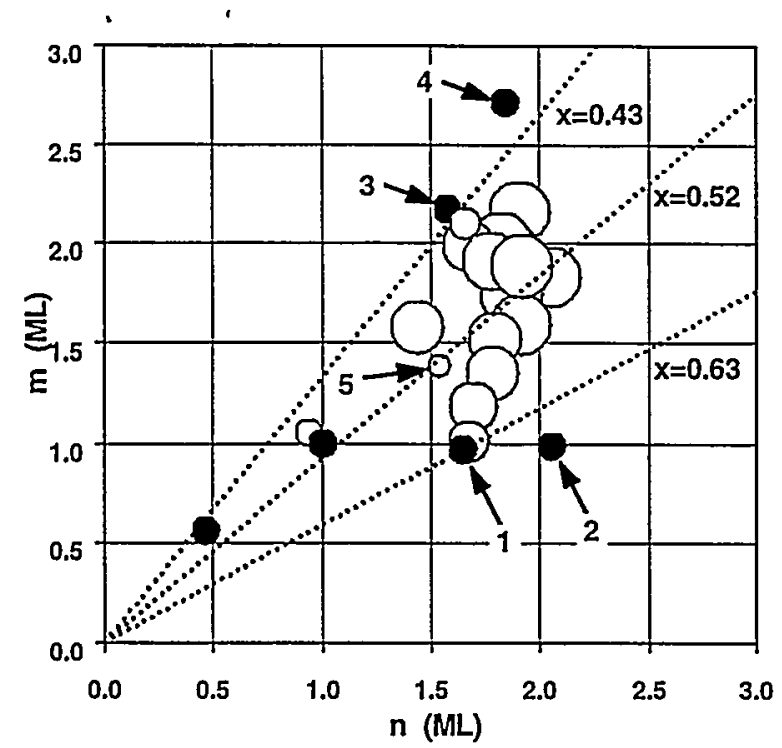

Figure 9. Plot of CM lateral satellite intensity ( $\propto$ area of open circles) versus layer thicknesses of the superlattice (InAs) ${ }_{n} /(\mathrm{AlAs})_{\mathrm{m}}$. Black circles represent near-zero intensity. Numbered points are discussed in the text. $\mathrm{X}$ is the average InAs mole fraction. 

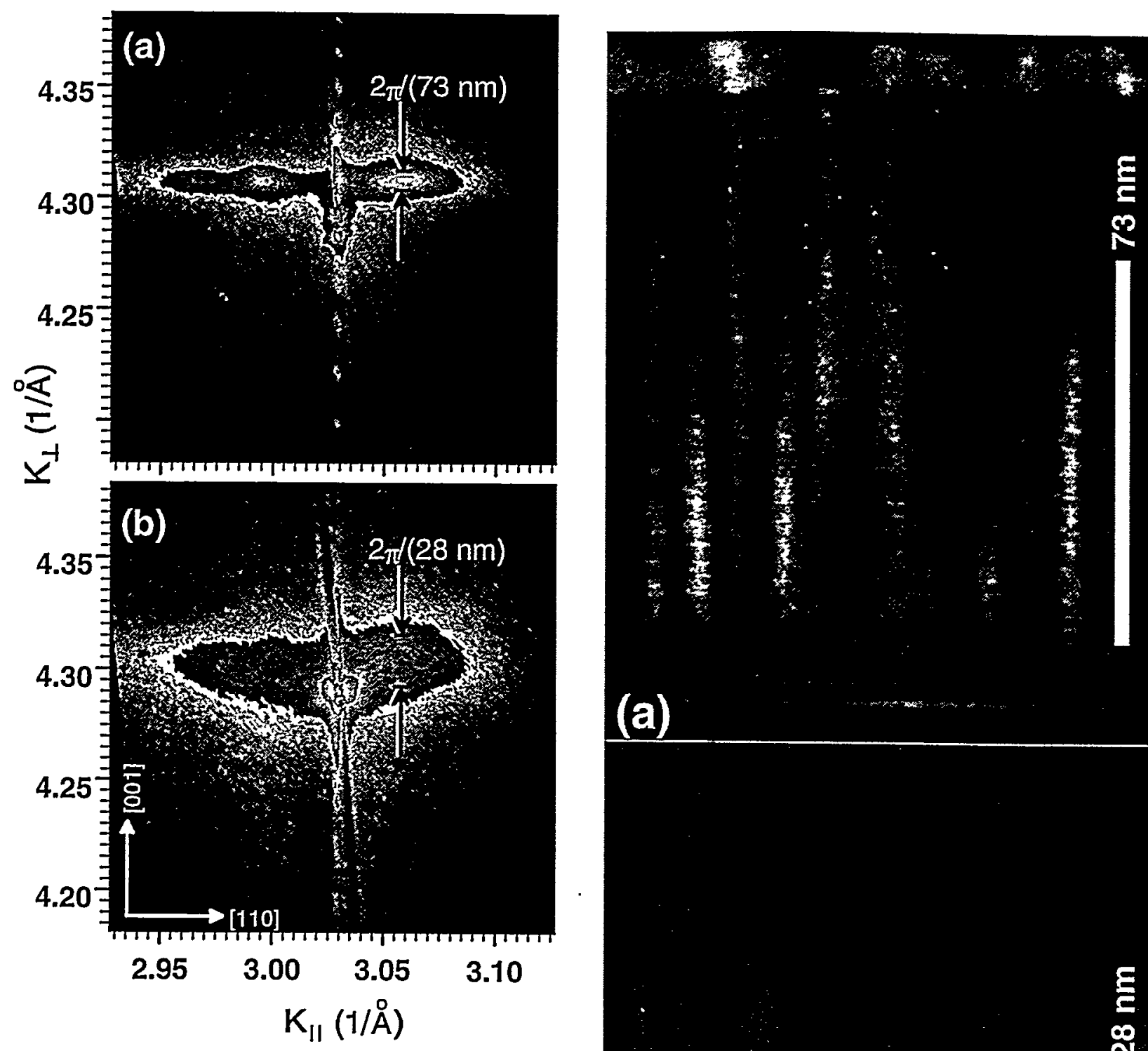

Figure 10 (above). XRD maps around (224) reflection and $\mathrm{CM}$ satellites for specimens a) grown at $0.50 \mathrm{ML} / \mathrm{s}$ (EA0187) and.b) grown at $0.18 \mathrm{ML} / \mathrm{s}$ (EA0186). Vertical domain height is indicated. The satellite in b) is more diffuse but has larger integrated intensity.

Figure 11 (right). Corresponding crosssectional TEM images for the specimens in Fig. 10, with domain height indicated.

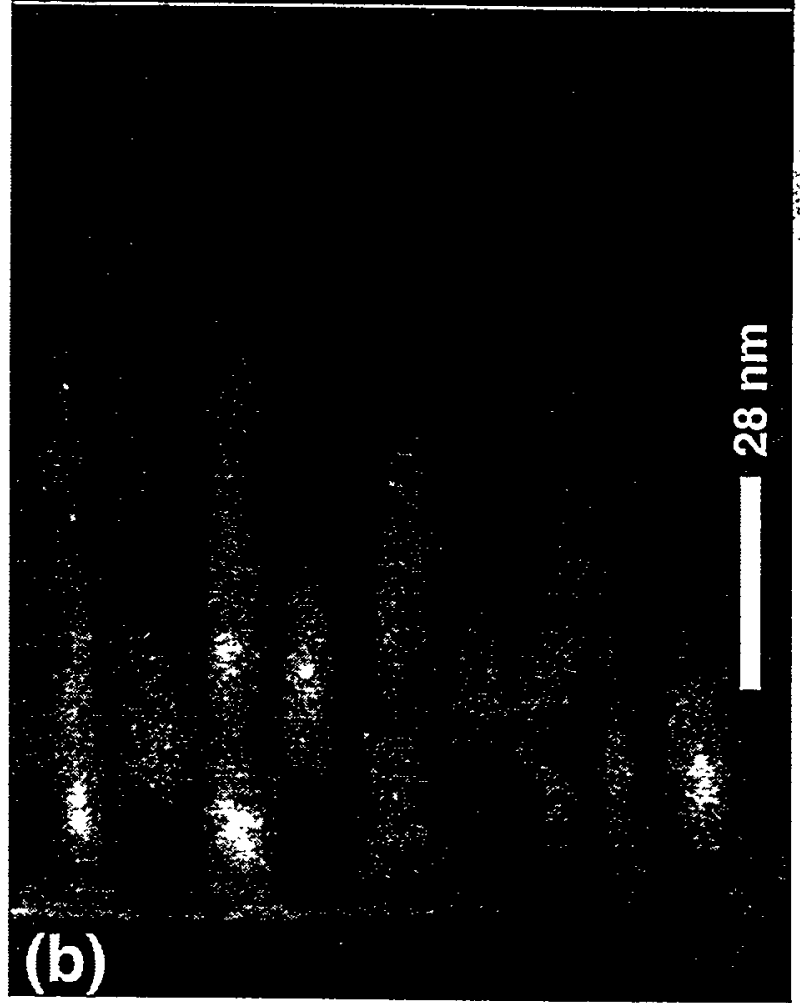



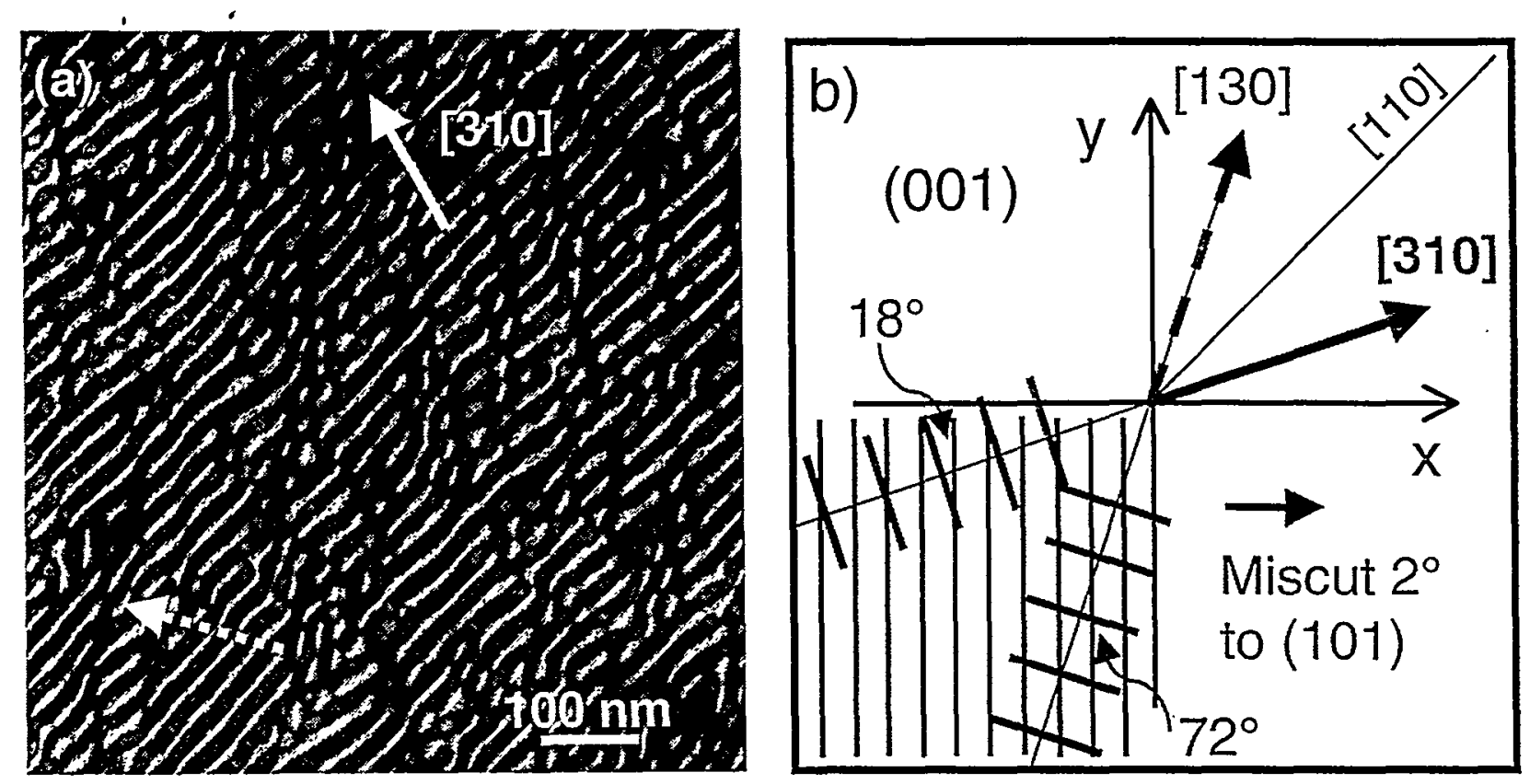

Figure 12. a) Plan-view, (020) dark-field TEM image of specimen miscut $2^{\circ}$ toward (101), with dominant [310] modulation in the growth plane. $\mathrm{n} \approx \mathrm{m} \approx 1.95$ (EA0478). b) Schematic of the (001) growth surface showing that the orientations of surface steps (long lines) resulting from miscut toward (101) are nearly parallel to wires (short lines) of the [310] modulation direction and near perpendicular to those of the [130] modulation direction. Image a) is rotated w.r.t. b). 


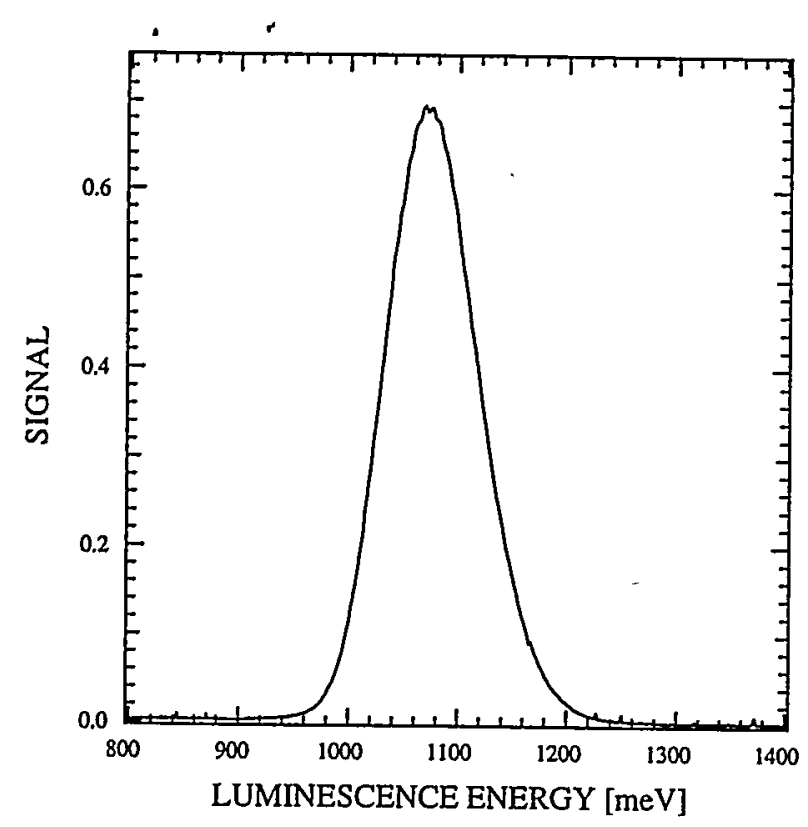

Figure 13. Low-temperature $(4.0 \mathrm{~K}) \mathrm{PL}$ spectrum from SPS with $\mathrm{n} \approx \mathrm{m} \approx 1.95$, and strong CM. Grown on InP substrate miscut $2^{\circ}$ toward (100) (EA0478). 